\title{
Comparative healing of swine skin following incisions with different surgical devices
}

\author{
Yuting Zhong ${ }^{1,2}$, Yufan Wei ${ }^{2,3}$, Ningning Min ${ }^{2,3}$, Qingyu Guan ${ }^{2,3}$, Jin Zhao ${ }^{2}$, Junyong Zhu ${ }^{2}$, Huayu $\mathrm{Hu}^{2,3}$, \\ Rui Geng ${ }^{1,2}$, Chenyan Hong ${ }^{2,3}$, Yashuang $\mathrm{Ji}^{2,3}$, Jie Li ${ }^{2}$, Yiqiong Zheng ${ }^{2}$, Yanjun Zhang ${ }^{2}$, Xiru $\mathrm{Li}^{2}$ \\ ${ }^{1}$ Medical School of Chinese PLA, Beijing, China; ${ }^{2}$ Department of General Surgery, Chinese People's Liberation Army General Hospital, Beijing, \\ China; ${ }^{3}$ School of Medicine, Nankai University, Tianjin, China \\ Contributions: (I) Conception and design: X Li, Y Zhang; (II) Administrative support: X Li; (III) Provision of study materials or patients: X Li; (IV) \\ Collection and assembly of data: All authors; (V) Data analysis and interpretation: All authors; (VI) Manuscript writing: All authors; (VII) Final \\ approval of manuscript: All authors. \\ Correspondence to: Xiru Li; Yanjun Zhang. Department of General Surgery, Chinese People's Liberation Army General Hospital, Beijing 100853, \\ China. Email: 2468li@sina.com; zhangyanjun301@163.com.
}

Background: Electrosurgical technology is widely used in surgical dissection and hemostasis, but the generated heat creates thermal injury to adjacent tissues and delays wound healing. The plasma blade (PB) applies pulsed radiofrequency (RF) to generate electrical plasma along the edge of a thin, flat, insulated electrode, minimizing collateral tissue damage. This study aimed to evaluate wound healing in swine skin following incision with a new surgical system that applies low-temperature plasma (NTS-100), a foreign PB, conventional electrosurgery (ES), and a scalpel blade.

Methods: In vitro porcine skin and an in vivo porcine skin model were used in this study. Full-thickness skin incisions $3 \mathrm{~cm}$ in length were made on the dorsum of each animal for each of the 5 surgical procedures at $0,21,28,35$, and 42 days. The timing of the surgical procedures allowed for wound-healing data points at $1,2,3$, and 6 weeks accordingly. Local operating temperature and blood loss were quantified. Wounds were harvested at designated time points, tested for wound tensile strength, and examined histologically for scar formation and tissue damage.

Results: Local operating temperature was reduced significantly with NTS-100 (cut mode $83.12 \pm 23.55^{\circ} \mathrm{C}$; coagulation mode $90.07 \pm 10.6{ }^{\circ} \mathrm{C}$ ) compared with $\mathrm{PB}$ (cut mode $94.46 \pm 11.48{ }^{\circ} \mathrm{C}$; coagulation mode $100.23 \pm 6.58{ }^{\circ} \mathrm{C}, \mathrm{P}<0.05$ ) and $\mathrm{ES}$ (cut mode $208.99 \pm 34.33{ }^{\circ} \mathrm{C}, \mathrm{P}<0.01$; coagulation mode $\left.233.37 \pm 28.69{ }^{\circ} \mathrm{C}, \mathrm{P}<0.01\right)$ in vitro. Acute thermal damage from NTS-100 was significantly less than ES incisions (cut mode: $247.345 \pm 42.274$ versus $495.295 \pm 103.525 \mu \mathrm{m}, \mathrm{P}<0.01$; coagulation mode: $351.419 \pm 127.948$ versus $584.516 \pm 31.708 \mu \mathrm{m}, \mathrm{P}<0.05)$. Bleeding, histological scoring of injury, and wound strength were equivalent for the NTS-100 and PB incisions.

Conclusions: The local operating temperature of NTS-100 was lower than PB, and NTS-100 had similarly reliable safety and efficacy.

Keywords: Plasma blade (PB); electrosurgery (ES); thermal damage; wound healing; scar formation

Submitted Jun 15, 2021. Accepted for publication Aug 05, 2021.

doi: $10.21037 /$ atm-21-3090

View this article at: https://dx.doi.org/10.21037/atm-21-3090

\section{Introduction}

Electrosurgery (ES) is used in over $80 \%$ of surgical operations (1), among which high-frequency electrotome (conventional ES) is currently the most widely used electrosurgical device (2). ES applies radiofrequency (RF) alternating current to generate heat at the cutting blade tip and tissue interface, allowing surgeons to effectively cut tissue and achieve rapid hemostasis simultaneously, resulting 


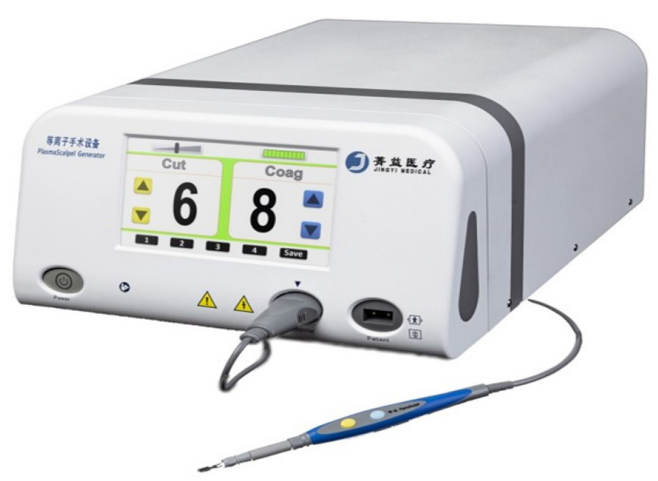

Figure 1 New-typed low temperature plasma (NTS-100) and its electrode.

in a shortened operation time and improved operational efficiency. The operating temperature reaches $200-300{ }^{\circ} \mathrm{C}$, and the generated heat creates thermal injury to adjacent tissues, causing delayed wound healing (3-5).

In recent years, the plasma blade $(\mathrm{PB})$ has been a breakthrough in the history of the development of electrosurgical surgery. It uses high-intensity RF pulses to induce the formation of electrical plasma along the edge of the electrode, limiting this effect on the tissue surface. Thus, $\mathrm{PB}$ reduces local operating temperature, creating less thermal damage to adjacent tissues (6-8). At present, this technology is widely used in surgical procedures abroad $(9,10)$. However, there has been inconsistent evidence regarding the comparative effectiveness of $\mathrm{PB}$ and $\mathrm{ES}$ in terms of the length of procedure time, drainage volume $(11,12)$, postoperative seroma $(11,12)$, and postoperative infection rates $(9,13,14)$. Therefore, more improvements should be made for better clinical effectiveness.

On this basis, our research team developed a new surgical system that applies low-temperature plasma (NTS-100) (Figure 1). This system also adopts pulsed RF energy, which improves the high-frequency generator, enhances the frequency of the main engine, and reduces the width of the slit of the surgical electrode to reduce thermal damage while enhancing hemostasis ability. To evaluate the efficacy and safety of NTS-100, we compared the incision healing of NTS-100 with ES and a foreign PB using an in vitro and in vivo porcine skin model. Local operating temperature and blood loss were quantified. Wounds were harvested at designated time points, tested for wound tensile strength, and examined histologically for scar formation and tissue damage. We present the following article in accordance with the ARRIVE reporting checklist (available at https:// dx.doi.org/10.21037/atm-21-3090).

\section{Methods}

\section{Infrared analysis of the instrument operating temperatures}

Infrared temperature images of the operation, which were conducted using NTS-100, PB (PEAK Surgical, Inc.), and ES (Valleylab Inc. USA), were captured by an infrared thermal imaging instrument (NS9300, NEC Corporation).

\section{Incisional wound model}

\section{In vitro experiment}

One piece of isolated pigskin with a size of $30 \times 20 \times 5$ $\mathrm{cm}$ was used. The experiment comprised six groups: (I) conventional electrosurgery on cut 40 mode (ESC), (II) conventional electrosurgery on coagulation 40 mode (ESCo), (III) the plasma blade on cut 6 mode (PBC), (IV) the plasma blade on coagulation 8 mode (PBCo), (V) new low-temperature plasma surgery system on cut 60 mode (NPC), (VI) new low-temperature plasma surgery system on coagulation 80 mode (NPCo). All 3 power settings had similar widths of thermal damage (data not shown).

\section{Porcine skin model}

The study was conducted on 6 healthy Bama miniature pigs (weight: $21-23 \mathrm{~kg}$; age: 10-13 months). Experimental animals were provided by Beijing Shichuangshiji Miniature Pig Breeding Base [SCXK (Beijing) 2013-0008] and were bred in the Animal Experiment Center of the First Medical Center of the Chinese PLA General Hospital. Animal experiments were performed under a project license (No. 20160010703) granted by the Ethics Committee of Chinese PLA General Hospital. All animal experiments complied with the National Institutes of Health Guide for the Care and Use of Laboratory Animals. The animals were acclimated for 1 week, and there was no abnormal situation. The experiment comprised 7 groups: the scalpel group (SC) and the above six groups.

\section{Surgical procedure}

\section{In vitro experiment}

Incisions were made on the isolated pigskin with 3 electrosurgical devices (NTS-100, PB, and ES) on cut and coagulation modes, as per the grouping and setting mode power described above. A total of 10 full-thickness skin 

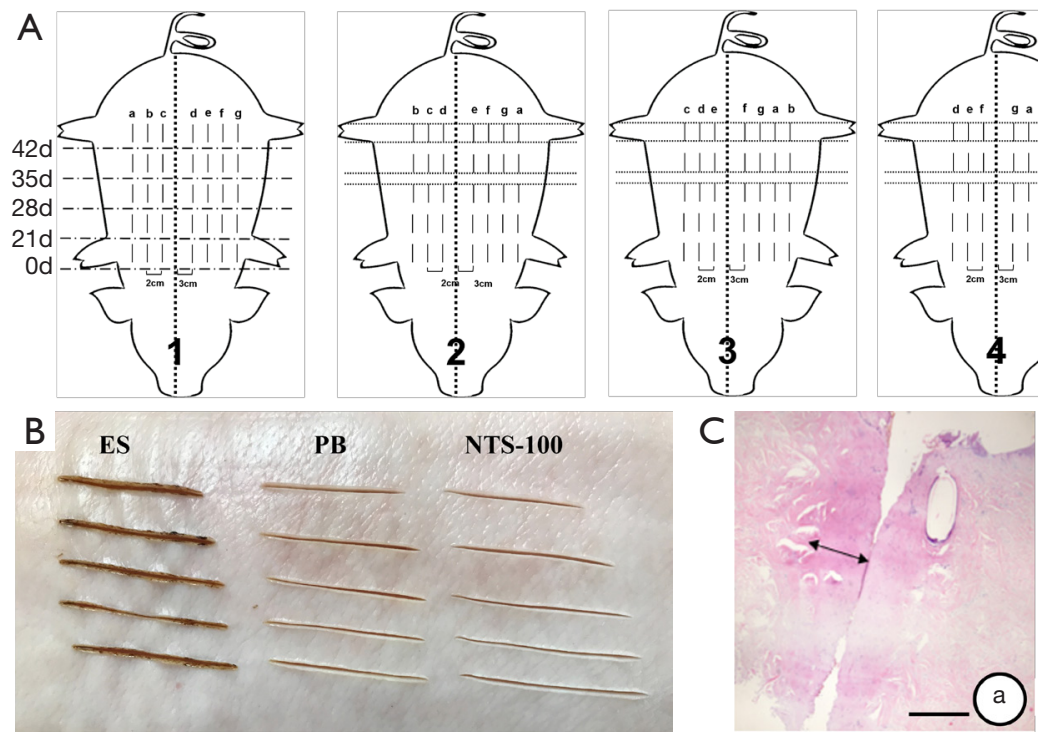
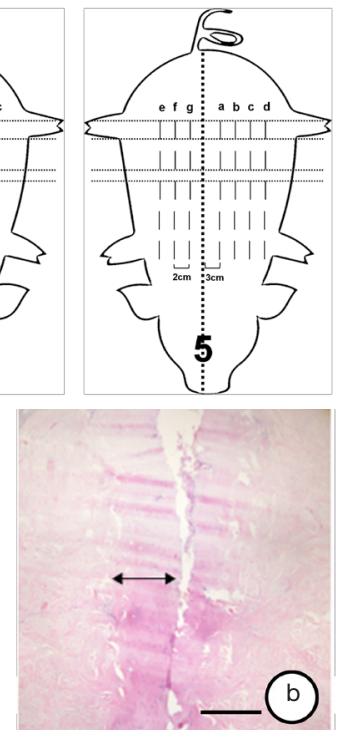

Figure 2 Incisional wound model. (A) Design of incisions (full-thickness skin incisions $3 \mathrm{~cm}$ in length $2 \mathrm{~cm}$ apart were made on the dorsum of each animal for each of the 5 surgical procedures at $0,21,28,35$, and 42 days. In order to prevent errors due to different skin thicknesses, the operation sequence of the skin incisions on the dorsum of the 6 animals was performed by 7 surgical modes in turn). (B) The appearance of the in vitro incisions made with ES, PB, and NTS-100, respectively. (C) Acute histology of the skin incisions produced by (a) ES, (b) PB, and (c) NTS-100, respectively. Arrows indicate the zone of coagulation necrosis. Acute thermal injury was determined as the maximum width of the zone of coagulation necrosis using hematoxylin and eosin sections. Scale bar: $0.5 \mathrm{~mm}$. ES, conventional electrosurgical instrument; PB, plasma blade; NTS-100, new surgical system which applies low temperature plasma.

incisions in each group were made at the same uniform speed.

\section{Porcine skin model}

Full-thickness skin incisions $3 \mathrm{~cm}$ in length were made on the dorsum of each pig for each of the 5 surgical procedures at $0,21,28,35$, and 42 days. The most medial incision was placed $3 \mathrm{~cm}$ from the dorsal spinal processes, with each subsequent incision placed $2 \mathrm{~cm}$ lateral to the prior incision, all in parallel orientation (Figure $2 A$ ). The surgical procedure at each time point consisted of 1 incision made with a scalpel and ES, PB, and NTS-100 cut and coagulation modes ( $\mathrm{n}=6$ in each group). All wounds were closed with a 3-0 nylon suture, covered with antibiotic ointment, and removed the sutures on day 14 . The timing of the surgical procedures allowed for wound-healing data points at 1,2, 3 , and 6 weeks, and the animals were sacrificed immediately after the surgical procedure on day 42 .

\section{Blood loss evaluation}

Filter paper used to absorb blood was weighed before and after surgery. Only 1 type of filter paper was used. After the incision operation of each instrument, a piece of sterile filter paper was immediately used to cover the incision for 1 minute until blood was completely absorbed on the filter paper during this period. Blood loss was measured by weighing the filter paper. No suction was used. The total weight during the operation of the blood filter paper was weighed by an electronic analysis balance (JA1003B, Shanghai Yueping Scientific Instrument Co., Ltd.).

\section{Histological examination}

A $2 \times 1 \mathrm{~cm}$ specimen containing the incision was excised and fixed in $10 \%$ buffered formalin for 24 hours and embedded in paraffin. Hematoxylin and eosin (HE) staining and immunohistochemistry were performed to observe the histomorphology. All specimens were coded and evaluated by light microscopy. Acute thermal injury (time point at week 0 ) was determined as the maximum width of the zone of coagulation necrosis using HE sections (15). All sections were examined for the width of the scar and the number of $\mathrm{T}$ lymphocytes and macrophages (time points at 1, 2, 3, 6 weeks). Images for measuring scar width were processed using Image J software (National Institutes of Health). The number of $T$ lymphocytes and macrophages were counted 
using the Image-Pro Plus software program.

\section{Wound strength}

A $2 \times 1 \mathrm{~cm}$ full-thickness strip of tissue with the incision at the midpoint was excised using a steel template. The wound burst strength of the tissues was measured using a biological force tester (MTS Bionix 858, MTS company).

\section{Statistical analysis}

The experimental data were statistically analyzed and processed by SPSS 17.0 software. The data were reported as mean \pm SEM. One-way analysis of variance (ANOVA) was used to compare the means of multiple groups. The value of $\mathrm{P}<0.05$ was considered statistically significant, and the value of $\mathrm{P}<0.01$ was considered highly statistically significant.

\section{Results}

\section{Instrument operating temperatures}

The color of isolated skin after the procedure changed obviously in the in vitro experiment. The incisions made by ES, PB, and NTS-100 were charred black, reddish-brown, and light yellow, respectively (Figure 2B). The difference in thermal damage was visible to the naked eye. The operating temperature of isolated pigskin with NTS-100 measured by an infrared camera was $83.12 \pm 23.55^{\circ} \mathrm{C}$ in cut mode and $90.07 \pm 10.6^{\circ} \mathrm{C}$ in coagulation mode, which were lower than ES $\left(208.99 \pm 34.33{ }^{\circ} \mathrm{C}\right.$ in cut mode and $233.37 \pm 28.69^{\circ} \mathrm{C}$ in coagulation mode, $\mathrm{P}<0.01)$ and the $\mathrm{PB}\left(100.23 \pm 6.58^{\circ} \mathrm{C}\right.$ in coagulation mode, $\mathrm{P}<0.05)(\mathrm{n}=10$ in each group).

\section{Blood loss evaluation}

NTS-100 system cut $(0.046 \pm 0.034 \mathrm{~g})$ and coagulation $(0.041 \pm 0.024 \mathrm{~g})$ modes demonstrated reduced bleeding compared with scalpel incisions $(0.410 \pm 0.291 \mathrm{~g}, \mathrm{P}<0.001)$, and there was no statistically significant difference compared with $\mathrm{PB}$ (cut mode $0.020 \pm 0.009 \mathrm{~g}$; coagulation mode $0.018 \pm 0.011 \mathrm{~g}$ ) and EC (cut mode $0.014 \pm 0.007 \mathrm{~g}$; coagulation mode $0.012 \pm 0.010 \mathrm{~g}$ ). The results indicated that NTS-100 had the same hemostatic effect as PB and ES.

\section{Zone of coagulation necrosis}

Acute thermal damage from NTS-100 (cut mode
$247.345 \pm 42.274 \mu \mathrm{m}$; coagulation mode $351.419 \pm$ $127.948 \mu \mathrm{m}$ ) was significantly less than ES incisions (cut mode 495.295 $\pm 103.525 \mu \mathrm{m}, \mathrm{P}=0.002$; coagulation mode $584.516 \pm 31.708 \mu \mathrm{m}, \mathrm{P}=0.012$ ), and there was no statistically significant difference compared with $\mathrm{PB}$ (cut mode $349.120 \pm 30.470 \mu \mathrm{m}$; coagulation mode $459.359 \pm 67.160 \mu \mathrm{m})$ (Figure 2C).

\section{Scar formation}

Histological scoring of injury with the NTS-100 was significantly less than ES (cut mode: $847.9250 \pm 146.943$ versus $1,806.617 \pm 254.372 \mu \mathrm{m}$ at 1 week, $\mathrm{P}<0.01$; $593.696 \pm 232.071$ versus $907.152 \pm 180.281 \mu \mathrm{m}$ at 2 weeks, $\mathrm{P}<0.05 ; 861.145 \pm 219.715$ versus $412.890 \pm 160.994 \mu \mathrm{m}$ at 3 weeks, $\mathrm{P}<0.05 ; 669.394 \pm 182.444$ versus $366.206 \pm$ $100.260 \mu \mathrm{m}$ at 6 weeks, $\mathrm{P}<0.05$; coagulation mode: $2,285.386 \pm 334.232$ versus $893.7277 \pm 151.942 \mu \mathrm{m}$ at 1 week, $\mathrm{P}<0.01 ; 1,591.094 \pm 254.284$ versus $830.904 \pm 143.469 \mu \mathrm{m}$ at 2 weeks, $\mathrm{P}<0.05 ; 1,075.201 \pm 490.276$ versus $728.059 \pm$ $127.176 \mu \mathrm{m}$ at 3 weeks; $722.071 \pm 167.581$ versus $392.539 \pm$ $81.264 \mu \mathrm{m}$ at 6 weeks, $\mathrm{P}<0.05)$, and there was no statistically significant difference compared with the PB (Figure 3).

\section{Inflammatory markers}

The distribution of T lymphocyte and macrophage immunohistochemical staining differed in each equipment group at each time point. Generally, the infiltration of $\mathrm{T}$ lymphocytes and macrophages for each device increased until the number reached the maximum at 1 week. After that, the infiltration at weeks 2,3 , and 6 gradually decreased with time.

The number of infiltrating $T$ lymphocytes and macrophages in the scalpel group was significantly lower than that in the other groups when the healing time was 1,2, and 3 weeks $(\mathrm{P}<0.05)$. At 6 weeks, there was no statistically significant difference between the scalpel group and the NTS-100 cutting mode group. As for the comparison of the 3 electrosurgical devices, at 1 and 2 weeks, there were no statistically significant differences among them. At 3 and 6 weeks, the incisions created by NTS-100 contained fewer T lymphocytes than ES incisions both in cut and coagulation mode (cut mode: $165.67 \pm 65.332$ versus $247.83 \pm 24.045$ at 3 weeks, $\mathrm{P}<0.01 ; 75.17 \pm 14.497$ versus $130.33 \pm 17.061$ at 6 weeks, $\mathrm{P}<0.01$; coagulation mode: $210.33 \pm 65.938$ versus $288.00 \pm 27.225$ at 3 weeks, $\mathrm{P}<0.01 ; 101.00 \pm 17.967$ versus $186.67 \pm 21.087$ at 6 weeks, $\mathrm{P}<0.01$ ) (Figure 4). 

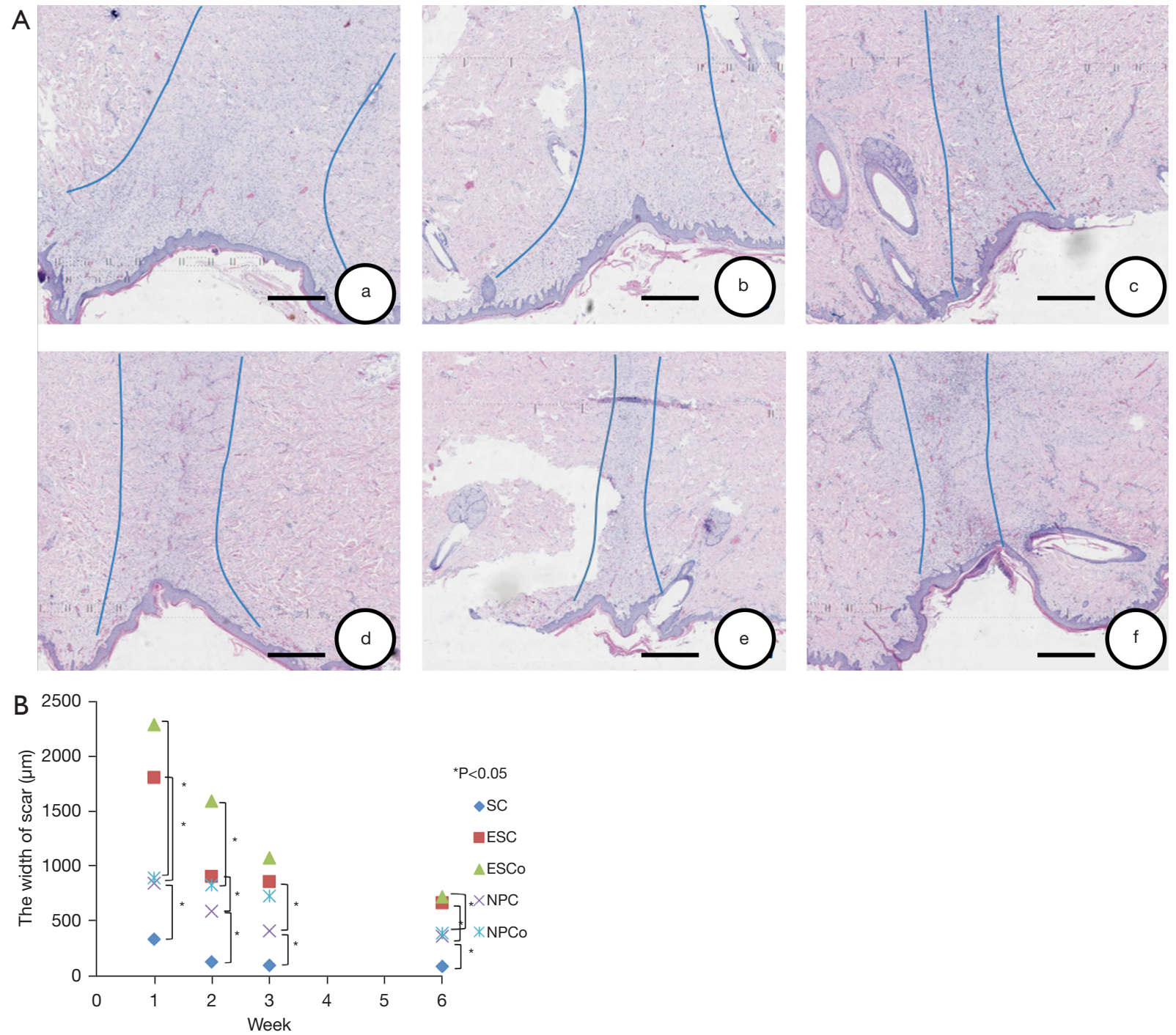

Figure 3 The examination of scar width. (A) Comparison of scar width at the same time after incision for (a) ESC, (b) ESCo, (c) PBC, (d) PBCo, (e) NPC, and (f) NPCo. All sections were stained with hematoxylin and eosin stain. All specimens were coded and evaluated by light microscopy $(100 \times)$ by a single pathologist in a blinded manner. Scale bar: $0.5 \mathrm{~mm}$. (B) The width of the scar in the wounds at $1,2,3$, and 6 weeks after incision ( ${ }^{*}$, compared with NTS-100). SC, scalpel; ES, conventional electrosurgical instrument; ESC, conventional electrosurgical instrument in cut mode; ESCo, conventional electrosurgical instrument in coagulation mode; PB, plasma blade; PBC, plasma blade in cut mode; PBCo, plasma blade in coagulation mode; NP, new surgical system which applies low temperature plasma; NPC, new surgical system which applies low temperature plasma in cut mode; NPCo, new surgical system which applies low temperature plasma in coagulation mode.

Likewise, at 3 and 6 weeks, the incisions created by NTS-100 contained fewer macrophages than ES incisions both in cut and coagulation mode (cut mode: $122.00 \pm 13.416$ versus $161.83 \pm 33.469$ at 3 weeks, $\mathrm{P}<0.01 ; 44.17 \pm 5.636$ versus $83.17 \pm 8.329$ at 6 weeks, $\mathrm{P}<0.01$; coagulation mode: $151.33 \pm 24.468$ versus $180.17 \pm 33.689$ at 3 weeks, $\mathrm{P}<0.01$; $79.33 \pm 9.331$ versus $107.83 \pm 9.109$ at 6 weeks, $\mathrm{P}<0.01$ ) (Figure 5). There was no significant difference between
NTS-100 and PB. The results indicated that NTS-100 had less infiltration of inflammatory cells in the late stage of skin healing, and the scar formation was better (Table 1).

\section{Wound burst strength evaluation}

Scalpel and NTS-100 incisions exhibited greater tensile strength compared with $\mathrm{PB}$ and ES incisions at all 

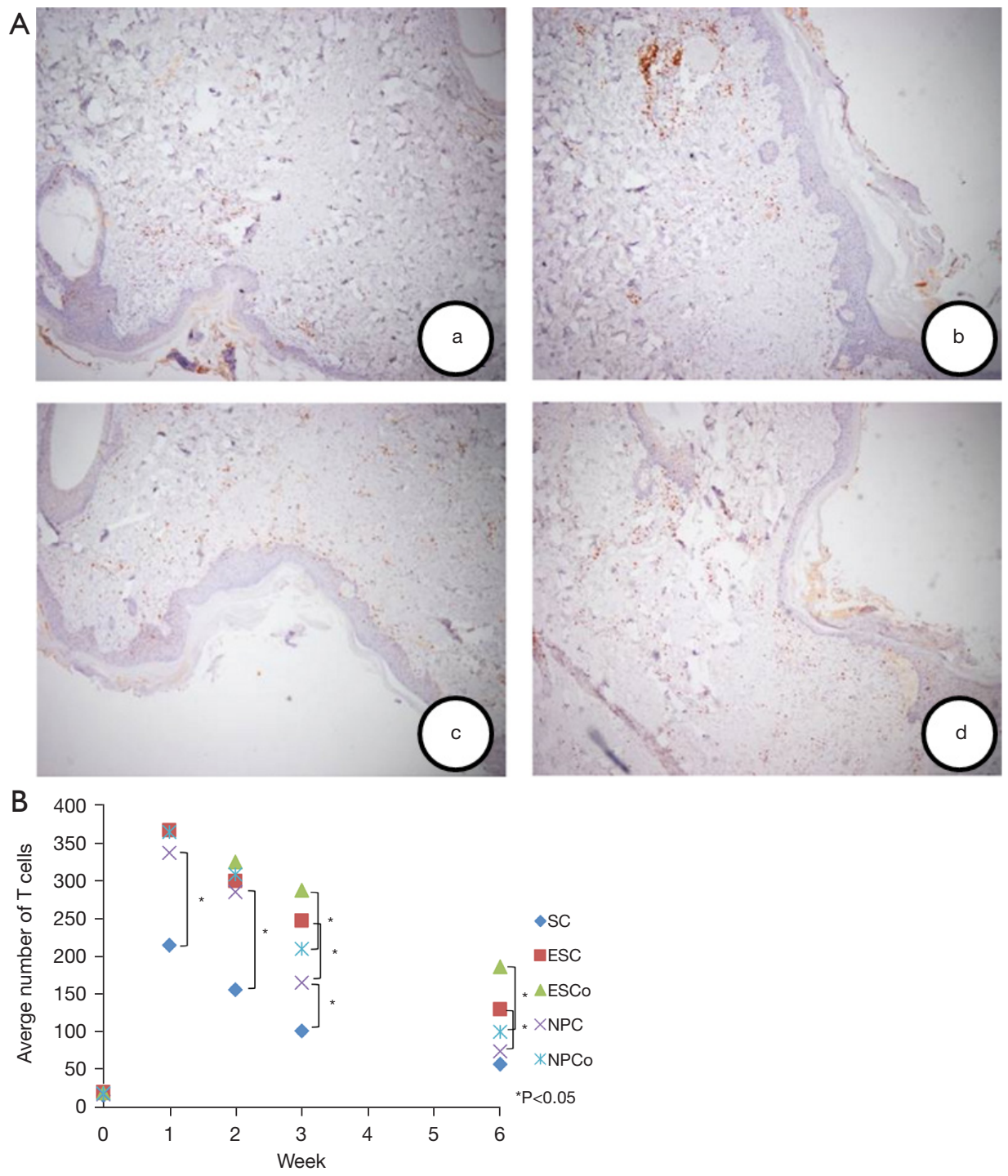

Figure 4 The examination of T lymphocytes. (A) Immunohistochemistry staining results of T lymphocytes by (a) scalpel, (b) ES, (c) PB, and (d) NTS-100, respectively. All sections were examined for T lymphocytes (CD3 M7254; Dako, Carpinteria, CA, USA). Sections were examined under high-power magnification $(100 \times)$ and the number of cells was counted by a blinded observer. (B) Number of T lymphocytes in the wounds at $0,1,2,3$, and 6 weeks after surgery (*, compared with NTS-100). SC, scalpel; ES, conventional electrosurgical instrument; ESC, conventional electrosurgical instrument in cut mode; ESCo, conventional electrosurgical instrument in coagulation mode; $\mathrm{PB}$, plasma blade; PBC, plasma blade in cut mode; PBCo, plasma blade in coagulation mode; NP, new surgical system which applies low temperature plasma; NPC, new surgical system which applies low temperature plasma in cut mode; NPCo, new surgical system which applies low temperature plasma in coagulation mode.

time points. By 3 weeks, the healed wound strength of NTS-100 (cut mode $59.52 \pm 13.36 \mathrm{~N}$, coagulation mode $68.91 \pm 22.40 \mathrm{~N}$ ) was stronger than ES (cut mode $30.39 \pm 16.68 \mathrm{~N}$, coagulation mode $68.91 \pm 22.40 \mathrm{~N}$ ), and the scar formation was better (Table 1, Figure 6).

\section{Safety evaluation}

During the NTS-100 operation, the animals had a stable heart rate and respiration. There was no unexpected morbidity and mortality, and the physiological condition of 

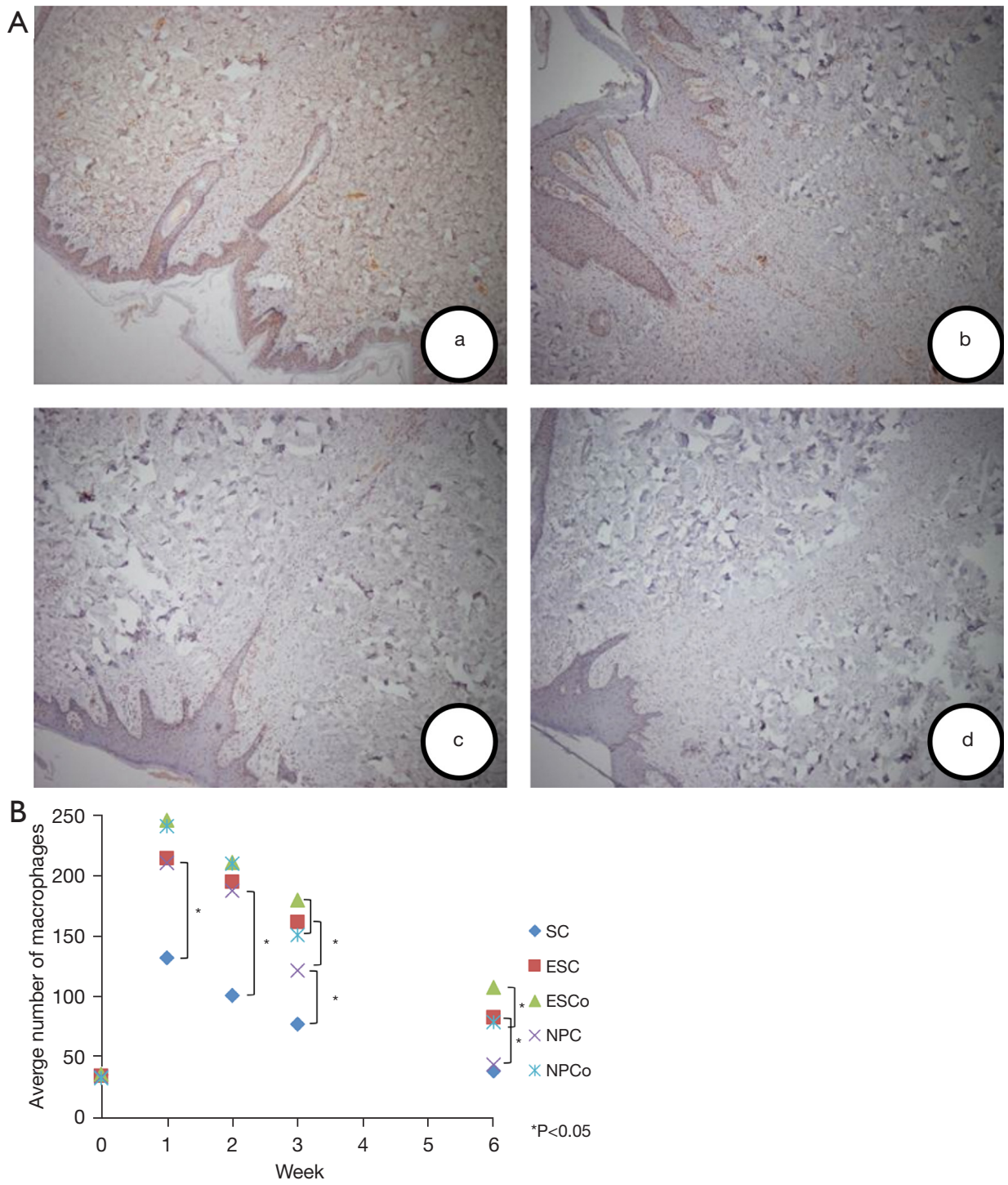

Figure 5 The examination of macrophages. (A) Immunohistochemistry results of macrophages by (a) scalpel, (b) ES, (c PB, and (d) NTS-100, respectively. All sections were examined for macrophages (CD68 NCL-L-CD68, Novacostra, Newcastle, UK). Sections were examined under high-power magnification (100x) and the number of cells was counted by a blinded observer. (B) Number of macrophages in the wounds at 0 , $1,2,3$, and 6 weeks after surgery ( ${ }^{*}$, compared with NTS-100). SC, scalpel; ES, conventional electrosurgical instrument; ESC, conventional electrosurgical instrument in cut mode; ESCo, conventional electrosurgical instrument in coagulation mode; PB, plasma blade; PBC, plasma blade in cut mode; PBCo, plasma blade in coagulation mode; NP, new surgical system which applies low temperature plasma; NPC, new surgical system which applies low temperature plasma in cut mode; NPCo, new surgical system which applies low temperature plasma in coagulation mode.

the experimental animals was normal, including heart rate, respiration, diet, and activities, both in intraoperative and postoperative anesthesia recovery. The NTS-100 surgical system had the same safety profile as other electrosurgical devices.

\section{Discussion}

Traditionally, the scalpel has been used as a surgical cutting tool due to the advantages of precision, tissue integrity preservation, and superior wound healing. However, bleeding limits its widespread use in procedures across 


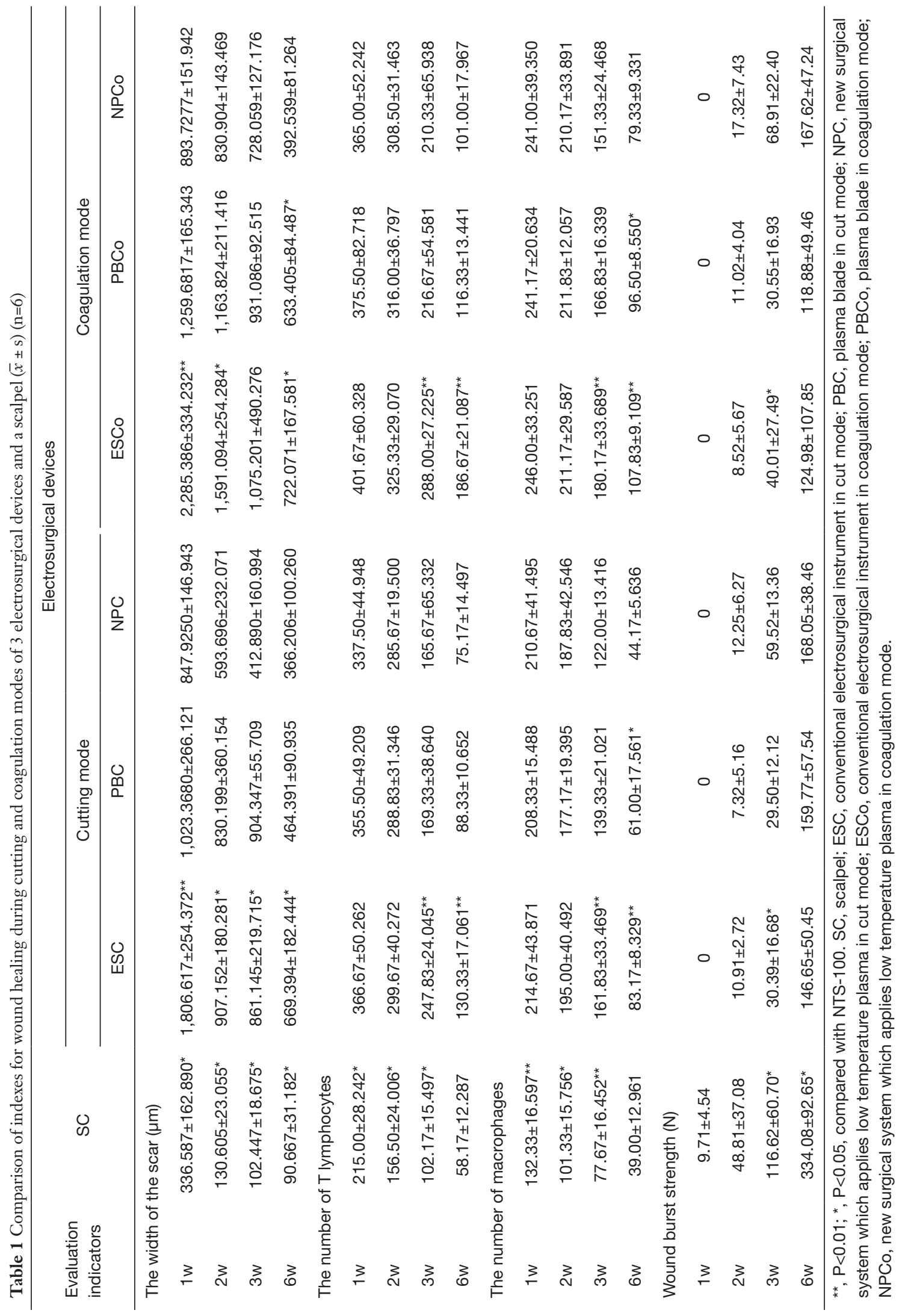




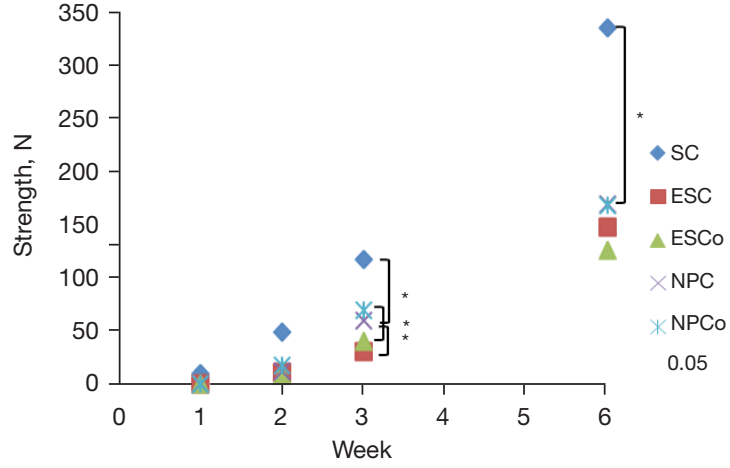

Figure 6 Wound burst strength at 1, 2, 3, and 6 weeks after surgery (*, compared with NTS-100). SC, scalpel; ES, conventional electrosurgical instrument; ESC, conventional electrosurgical instrument in cut mode; ESCo, conventional electrosurgical instrument in coagulation mode; $\mathrm{PB}$, plasma blade; $\mathrm{PBC}$, plasma blade in cut mode; PBCo, plasma blade in coagulation mode; NP, new surgical system which applies low temperature plasma; NPC, new surgical system which applies low temperature plasma in cut mode; NPCo, new surgical system which applies low temperature plasma in coagulation mode.

surgical specialties, as hemostatic control and dissection capability are fundamental to the practice of surgery $(7,16)$. Consequently, numerous electrosurgical devices have been explored to ensure the basic functions of cutting and coagulation $(2,17-19)$. Although hemostasis can be achieved, electrosurgical devices induce thermal damage to adjacent tissues, inferior wound healing, and a poor cosmetic effect, limiting their applications $(20,21)$. A thorough understanding of these technologies and their applications and the potential risks and the appropriate settings can help improve the effectiveness and safety of surgery. For example, in neurosurgery, the application of ES is limited, which may cause acute and subacute nerve damage. In thyroid surgery, the thermal damage induced by electrosurgical equipment may cause damage to the recurrent laryngeal nerves, resulting in dysphonia or even potentially life-threatening asphyxia (22-24).

The PB is a relatively new electrosurgical technology developed to achieve cutting and coagulation effects with a relatively low operating temperature, which reduces thermal damage $(6,25)$. It applies high-frequency RF pulses to generate electrical plasma along the edge of the electrode (26). Several studies have shown that this technology allows for the precise dissection of rabbit, bovine, and human retinas and lens capsules, even when completely submerged in a liquid medium (27-30). The PB system has been approved by the Food and Drug Administration (FDA) for use in the cutting and coagulation of soft tissues during otolaryngology surgical procedures. A prospective double-blinded randomized controlled trial showed that $\mathrm{PB}$ tonsillectomy achieved a faster postoperative recovery in terms of the time taken to achieve pain-free swallowing (31). However, no significant differences were found between $\mathrm{PB}$ and ES regarding postoperative bleeding $(11,32,33)$, postoperative hematoma $(9,11,14)$, and inflammatory responses (13). Therefore, more improvements should be made for better clinical effectiveness.

On this basis, our research team developed a new surgical system that applies low-temperature plasma (NTS-100). This system also adopts pulsed RF energy, which improves the high-frequency generator, enhances the frequency of the main engine, and reduces the width of the slit of the surgical electrode to reduce the thermal damage while enhancing hemostasis ability. This study investigated operating temperature, blood loss, thermal injury depth, inflammation, and scarring in swine skin by comparing the histology of incisions made with NTS-100, PB, ES, and a scalpel.

The local operating temperature is related to the thermal injury of tissue and the thermal diffusion. Except for thermal injury, the heated tissues may adhere to the hot electrode. Tissue sticking impairs the accuracy and efficiency of the operation process (34). Therefore, operating temperature control is essential to improve instrument performance, reduce tissue sticking, and prevent thermal injury. Our study showed that the operating temperature of isolated pig skin with NTS-100 was much lower than ES in cut mode and coagulation mode and lower than PB. It indicated that NTS-100 had a better performance in preventing thermal damage and reducing tissue sticking. Moreover, the hemostasis effect was comparable among these three devices, indicating that despite the lower working temperature and less thermal damage to tissues of NTS-100, high operating temperature and deep thermal coagulum associated with ES are not essential for hemostasis. Further studies on this effect should be conducted.

Wound healing is one of the most complex processes in the human body, which involves various cell types in the phases of hemostasis, inflammation, growth, reepithelialization, and remodeling (35). Several studies have shown that inflammatory cells are recruited and activated in the early stage of injury to defend against bacteria and 
combat self and foreign antigens. As the inflammatory phase ends, angiogenesis occurs, and granulation tissue forms, shifting the wound microenvironment from the inflammatory to the growth state (36-38). However, increased inflammation can alter the outcome of wound repair. $\mathrm{T}$ lymphocytes and macrophages are critical to normal wound healing and tissue regeneration (39-42), so to some extent, the degree of infiltration of $\mathrm{T}$ lymphocytes and macrophages can reflect the healing of wounds.

At each designated time point, scar formation was less on the incisions of NTS-100 than on that of ES. At 3 and 6 weeks, the incisions created by NTS-100 contained fewer $\mathrm{T}$ lymphocytes and macrophages than incisions created by ES both in cut and coagulation mode. The infiltration of $\mathrm{T}$ lymphocytes and macrophages and the burst strength of the incisions created by the NTS-100 and PB were similar. These results indicate that the NTS-100 created less scar formation and reduced inflammation, which might be associated with lower local working temperature and less tissue thermal damage. The levels of $\mathrm{T}$ lymphocytes and macrophages were higher in the NTS-100 and PB groups than in the scalpel group, and the wound burst strength was lower in the NTS-100 and PB groups than in the scalpel group. The greater infiltration by $\mathrm{T}$ lymphocytes and macrophages during the course of healing in the ES group is consistent with previous research results showing that abnormal inflammation is associated with reduced wound burst strength (8).

Wound healing in adults results in a fibrotic scar resulting from differential cellular responses towards mechanical stress within the healing skin, but excessive scarring shifts the balance towards fibrotic states of hypertrophic scarring and keloid formation $(43,44)$. The appearance of scars was strongly related to thermal damage and wound histological examination (31). Histological examinations showed reduced scar width in the NTS-100 and PB groups compared with the ES group, which indicated that increased inflammation influences wound repair. The incisions by NTS-100 can achieve a better cosmetic effect. Further study of scar formation is necessary, especially in the field of plastic and reconstructive surgery.

Furthermore, additional benefits of the NTS-100 were observed during experiments. Heated electrodes are associated with surgical smoke, which is harmful to surgeons and may affect an operation by interfering with the surgeon's visibility $(45,46)$. The difference in smoke production and blade eschars was visible to the naked eye. NTS-100 produced significantly less surgical smoke than ES, and the blade was free of eschar, resulting in better surgical fluency. The reason may be related to the plasma at the edge of the blade. Compared with ES, the plasma prevents burning tissue at a high temperature, which produces smoke and forms eschars. Therefore, follow-up research will focus on determining the content of particulate matter in surgical smoke, the composition of the smoke, especially the composition and content of harmful substances, and find suitable indicators to objectively evaluate and compare the sharpness of the blade and the fluency of the operation. Consequently, NTS-100 tries to reduce the occupational exposure of medical staff and make the operation process more efficient at the same time (47).

Nowadays, NTS-100 has already been used in breast surgery, general surgery, and head and neck surgery, and clinical data have been collected to verify its clinical effectiveness. We believe that as an innovative electrosurgical device with a relatively low operating temperature, our surgical system, which applies lowtemperature plasma (NTS-100), has a promising future and will be widely used in surgical procedures. Then, more clinical data will be compared with current electrosurgical devices (10) to improve our system continuously.

\section{Conclusions}

The local operating temperature of NTS-100 was lower than PB, and NTS-100 had similarly reliable safety and efficacy.

\section{Acknowledgments}

Funding: This work was supported by Translational Medicine Project of PLA General Hospital (grant No. 2016TM-006).

\section{Footnote}

Reporting Checklist: The authors have completed the ARRIVE reporting checklist. Available at https://dx.doi. org/10.21037/atm-21-3090

Data Sharing Statement: Available at https://dx.doi. org/10.21037/atm-21-3090

Peer Review File: Available at https://dx.doi.org/10.21037/ atm-21-3090 
Conflicts of Interest: All authors have completed the ICMJE uniform disclosure form (available at https://dx.doi. org/10.21037/atm-21-3090). The authors have no conflicts of interest to declare.

Ethical Statement: The authors are accountable for all aspects of the work in ensuring that questions related to the accuracy or integrity of any part of the work are appropriately investigated and resolved. Animal experiments were performed under a project license (No. 20160010703) granted by the Ethics Committee of Chinese PLA General Hospital, in compliance with the NIH guidelines for the care and use of animals.

Open Access Statement: This is an Open Access article distributed in accordance with the Creative Commons Attribution-NonCommercial-NoDerivs 4.0 International License (CC BY-NC-ND 4.0), which permits the noncommercial replication and distribution of the article with the strict proviso that no changes or edits are made and the original work is properly cited (including links to both the formal publication through the relevant DOI and the license). See: https://creativecommons.org/licenses/by-ncnd/4.0/.

\section{References}

1. Meeuwsen F, Guédon A, Klein J, et al. Electrosurgery: short-circuit between education and practice. Minim Invasive Ther Allied Technol 2019;28:247-53.

2. Palanker DV, Vankov A, Huie P. Electrosurgery with cellular precision. IEEE Trans Biomed Eng 2008;55:838-41.

3. Massarweh NN, Cosgriff N, Slakey DP. Electrosurgery: history, principles, and current and future uses. J Am Coll Surg 2006;202:520-30.

4. Wu MP, Ou CS, Chen SL, et al. Complications and recommended practices for electrosurgery in laparoscopy. Am J Surg 2000;179:67-73.

5. Blankenship ML. Physical modalities. Electrosurgery, electrocautery and electrolysis. Int J Dermatol 1979;18:443-52.

6. Schlosshauer T, Kiehlmann M, Riener MO, et al. Comparative analysis on the effect of low-thermal plasma dissection device (PEAK PlasmaBlade) vs conventional electrosurgery in post-bariatric abdominoplasty: A retrospective randomised clinical study. Int Wound J 2019;16:1494-502.
7. Charoenkwan K, Iheozor-Ejiofor Z, Rerkasem $\mathrm{K}$, et al. Scalpel versus electrosurgery for major abdominal incisions. Cochrane Database Syst Rev 2017;6:CD005987.

8. Loh SA, Carlson GA, Chang EI, et al. Comparative healing of surgical incisions created by the PEAK PlasmaBlade, conventional electrosurgery, and a scalpel. Plast Reconstr Surg 2009;124:1849-59.

9. Kypta A, Blessberger H, Saleh K, et al. An electrical plasma surgery tool for device replacement--retrospective evaluation of complications and economic evaluation of costs and resource use. Pacing Clin Electrophysiol 2015;38:28-34.

10. Peprah K, Spry C. CADTH Rapid Response Reports. Pulsed Electron Avalanche Knife (PEAK) PlasmaBlade versus Traditional Electrocautery for Surgery: A Review of Clinical Effectiveness and Cost-Effectiveness. Ottawa $(\mathrm{ON})$ : Canadian Agency for Drugs and Technologies in Health Copyright (C 2019 Canadian Agency for Drugs and Technologies in Health.; 2019.

11. Duscher D, Aitzetmüller MM, Shan JJ, et al. Comparison of Energy-Based Tissue Dissection Techniques in Abdominoplasty: A Randomized, OpenLabel Study Including Economic Aspects. Aesthet Surg J 2019;39:536-43.

12. Sowa $Y$, Inafuku N, Kodama T, et al. Preventive Effect on Seroma of Use of PEAK PlasmaBlade after Latissimus Dorsi Breast Reconstruction. Plast Reconstr Surg Glob Open 2018;6:e2035.

13. Marangi GF, Pallara T, Lamberti D, et al. An electrical plasma dissection tool for surgical treatment of chronic ulcers: Results of a prospective randomised trial. Int Wound J 2018;15:717-21.

14. Kypta A, Blessberger H, Kammler J, et al. Economic assessment of traditional surgical intervention versus use of a new innovative radiofrequency based surgical system in device replacements. PLoS One 2018;13:e0192587.

15. Lacitignola L, Desantis S, Izzo G, et al. Comparative Morphological Effects of Cold-Blade, Electrosurgical, and Plasma Scalpels on Dog Skin. Vet Sci 2020;7:8.

16. Charoenkwan K, Chotirosniramit N, Rerkasem K. Scalpel versus electrosurgery for abdominal incisions. Cochrane Database Syst Rev 2012;(6):CD005987.

17. Pogorelić Z, Perko Z, Druzijanić N, et al. How to prevent lateral thermal damage to tissue using the harmonic scalpel: experimental study on pig small intestine and abdominal wall. Eur Surg Res 2009;43:235-40. 
18. Huang TY, Lin YC, Tseng HY, et al. Safety of Ligasure exact dissector in thyroidectomy with continuous neuromonitoring: a porcine model. Gland Surg 2020;9:702-10.

19. Hambley R, Hebda PA, Abell E, et al. Wound healing of skin incisions produced by ultrasonically vibrating knife, scalpel, electrosurgery, and carbon dioxide laser. J Dermatol Surg Oncol 1988;14:1213-7.

20. Taheri A, Mansoori P, Sandoval LF, et al. Electrosurgery: part I. Basics and principles. J Am Acad Dermatol 2014;70:591.e1-e14.

21. Taheri A, Mansoori P, Sandoval LF, et al. Electrosurgery: part II. Technology, applications, and safety of electrosurgical devices. J Am Acad Dermatol 2014;70:607. e1-e12.

22. Chen C, Kallakuri S, Cavanaugh JM, et al. Acute and subacute effects of the ultrasonic blade and electrosurgery on nerve physiology. Br J Neurosurg 2015;29:569-73.

23. Yang X, Cao J, Yan Y, et al. Comparison of the safety of electrotome, Harmonic scalpel, and LigaSure for management of thyroid surgery. Head Neck 2017;39:1078-85.

24. Hayami M, Watanabe M, Mine S, et al. Lateral thermal spread induced by energy devices: a porcine model to evaluate the influence on the recurrent laryngeal nerve. Surg Endosc 2019;33:4153-63.

25. Zientara A, Komminoth P, Seifert B, et al. Skeletonized internal thoracic artery harvesting: a low thermal damage electrosurgical device provides improved endothelial layer and tendency to better integrity of the vessel wall compared to conventional electrosurgery. J Cardiothorac Surg 2018;13:105.

26. Tan A, Ganhasan S, Lu P, et al. PEAK PlasmaBlade versus monopolar electrocautery tonsillectomy in adults: A prospective double-blinded randomized controlled trial. Am J Otolaryngol 2019;40:478-81.

27. Palanker DV, Miller JM, Marmor MF, et al. Pulsed electron avalanche knife (PEAK) for intraocular surgery. Invest Ophthalmol Vis Sci 2001;42:2673-8.

28. Palanker D, Nomoto H, Huie P, et al. Anterior capsulotomy with a pulsed-electron avalanche knife. J Cataract Refract Surg 2010;36:127-32.

29. Priglinger SG, Haritoglou C, Palanker DV, et al. Pulsed electron avalanche knife (PEAK-fc) for dissection of retinal tissue. Arch Ophthalmol 2005;123:1412-8.

30. Priglinger SG, Palanker D, Alge CS, et al. Pulsed electron avalanche knife: new technology for cataract surgery. Br J Ophthalmol 2007;91:949-54.
31. Martin P. Wound healing--aiming for perfect skin regeneration. Science 1997;276:75-81.

32. Lane JC, Dworkin-Valenti J, Chiodo L, et al. Postoperative tonsillectomy bleeding complications in children: A comparison of three surgical techniques. Int J Pediatr Otorhinolaryngol 2016;88:184-8.

33. Thottam PJ, Christenson JR, Cohen DS, et al. The utility of common surgical instruments for pediatric adenotonsillectomy. Laryngoscope 2015;125:475-9.

34. Ou KL, Chu JS, Hosseinkhani H, et al. Biomedical nanostructured coating for minimally invasive surgery devices applications: characterization, cell cytotoxicity evaluation and an animal study in rat. Surg Endosc 2014;28:2174-88.

35. Rodrigues M, Kosaric N, Bonham CA, et al. Wound Healing: A Cellular Perspective. Physiol Rev 2019;99:665-706.

36. Wilgus TA, Roy S, McDaniel JC. Neutrophils and Wound Repair: Positive Actions and Negative Reactions. Adv Wound Care (New Rochelle) 2013;2:379-88.

37. Davies LC, Jenkins SJ, Allen JE, et al. Tissue-resident macrophages. Nat Immunol 2013;14:986-95.

38. Werner S, Krieg T, Smola H. Keratinocyte-fibroblast interactions in wound healing. J Invest Dermatol 2007;127:998-1008.

39. Zhu Z, Ding J, Ma Z, et al. Systemic depletion of macrophages in the subacute phase of wound healing reduces hypertrophic scar formation. Wound Repair Regen 2016;24:644-56.

40. Hu MS, Walmsley GG, Barnes LA, et al. Delivery of monocyte lineage cells in a biomimetic scaffold enhances tissue repair. JCI Insight 2017;2:96260.

41. Tanno H, Kawakami K, Kanno E, et al. Invariant NKT cells promote skin wound healing by preventing a prolonged neutrophilic inflammatory response. Wound Repair Regen 2017;25:805-15.

42. Wong VW, Paterno J, Sorkin M, et al. Mechanical force prolongs acute inflammation via T-celldependent pathways during scar formation. FASEB J 2011;25:4498-510.

43. Walmsley GG, Maan ZN, Wong VW, et al. Scarless wound healing: chasing the holy grail. Plast Reconstr Surg 2015;135:907-17.

44. Gurtner GC, Werner S, Barrandon Y, et al. Wound repair and regeneration. Nature 2008;453:314-21.

45. Al Sahaf OS, Vega-Carrascal I, Cunningham FO, et al. Chemical composition of smoke produced by highfrequency electrosurgery. Ir J Med Sci 2007;176:229-32. 
46. Ulmer BC. The hazards of surgical smoke. AORN J 2008;87:721-34; quiz 735-8.

47. Tani T, Naka S, Tani S, et al. The invention of microwave surgical scissors for seamless coagulation and cutting.

Cite this article as: Zhong Y, Wei Y, Min N, Guan Q, Zhao J, Zhu J, Hu H, Geng R, Hong C, Ji Y, Li J, Zheng Y, Zhang Y, Li $\mathrm{X}$. Comparative healing of swine skin following incisions with different surgical devices. Ann Transl Med 2021;9(20):1514. doi: 10.21037/atm-21-3090
Surg Today 2018;48:856-64.

(English Language Editors: C. Betlazar-Maseh and J. Chapnick) 